\title{
AU PAYS DE(S) INTÉGRÉ(S) DE GABRIEL KUITCHE FONKOU : DE LA PROBLÉMATIQUE DE L'INTÉGRATION BELLICISTE À LA RECONSTRUCTION IDENTITAIRE
}

VOKENG NGNINTEDEM, Guilioh Merlain ${ }^{1}$

RÉSUMÉ: Le roman de Gabriel Kuitche Fonkou laisse découvrir un univers manichéen dans lequel interagissent les cultures bamiléké, fang-béti et autres. Dans un tel contexte, l'espace multiculturel devient le théâtre d'un champ de positionnement; ce qui complexifie la coexistence entre les cultures et les peuples. Cela signifie que l'abolition des distances culturelles ne rend pas toujours faciles la compréhension entre les peuples et les relations entre groupes ethniques. Au Pays de(s) intégré( $s$ ) met en scène les enjeux et logiques identitaires. Ainsi, Kuitche Fonkou révèle le caractère conflictuel et ambivalent de ces lieux identitaires et montre la complexité des relations sociales qui jouent à la croisée des appartenances ethniques et socioéconomiques dans un pays où l'intégration nationale est considérée comme le facteur principal d'unité. En nous servant des trois modèles de sociétés multiculturelles proposés par Lüsebrink et en prenant appui sur la notion d' «ethnicisation » théorisée par David Simo, cet

${ }^{1}$ Université de Maroua/École Normale Supérieure, Chargé de Cours, Enseignant-Chercheur, gvokeng@yahoo.fr 
article vise à montrer que chez Kuitche Fonkou, l'ethnie est instrumentalisée et devient l'objet principal de la loyauté, le but et le moyen de toute action. Il apparaîtra au final que ce professeur-écrivain camerounais cherche à fabriquer un modèle culturel et identitaire qui brise les barrières ethnico-tribales.

MOTS-CLÉS : Altérité, Culture, Ethnie, Identité, Intégration, Multiculturalisme, Tribu.

\title{
GABRIEL KUITCHE FONKOU'S AU PAYS DE(S) INTÉGRÉ(S): FROM THE ISSUE OF BELLICOSE INTEGRATION TO THE RECONSTRUCTION OF IDENTITY
}

\begin{abstract}
Gabriel Kuitche Fonkou's novel reveals a Manichean universe in which Bamileke, Fang-Beti and other cultures interact. In such a context, the multicultural space becomes the theater of a positioning field; which complicates coexistence between cultures and peoples. This means that the abolition of cultural distances does not make easy the understanding between peoples and relations between ethnic groups. Au Pays de(s) intégré(s) puts in scene the stakes and logic of identity. Thus, Kuitche Fonkou reveals the conflicting and ambivalent nature of these identity places and shows the complexity of social relations that play at the crossroads of membership ethnic and socio-economic in a country where national integration is considered as the main factor of unit. By using the three models of multicultural societies proposed by Lüsebrink and drawing on the notion of "ethnicization" theorized by David Simo, this article aims to show that at Kuitche Fonkou, ethnicity is instrumentalized and becomes the main object of loyalty, the goal and the means of any action. It will appear in the end that this Cameroonian professor-writer seeks to fabricate a cultural and identity model that breaks the ethnico-tribal barriers.
\end{abstract}

KEYWORDS: Otherness, Culture, Ethnic group, Identity, Integration, Multiculturalism, Tribe.

\section{INTRODUCTION}

Au pays de(s)intégré(s) de Gabriel K. Fonkou met en exergue les différentes types de discrimination tribale et/ou régionaliste et leurs conséquences sociales, politiques, économiques et culturelles. Dans ce roman, la question de la construction nationale se heurte à la manipulation subtile des particularités ethniques et tribales. Ainsi, l'instrumentalisation des diversités ethniques et tribales à des fins politiques n'est pas 
seulement une sérieuse menace pour l'émergence véritable d'une nation, mais aussi et surtout un poison psychosociologique hautement nocif et capable de détruire gravement la personnalité des citoyens. C'est dans cette perspective que Marc Angenot stipule que

le ressentiment n'est pas engendré sui generis dans des groupes humains prédisposés par leur histoire. Il se développe dans des états de société qui, à force de déstabiliser leurs membres, les priver de repères, les étourdir de mensonges et de simulacres, obscurcir le sens du monde et les valeurs collectives, entretenir des conflits endémiques, stimulent le ressentiment de tous et de chacun, incitent à trouver des anesthésiques face aux frustrations et aux douleurs qu'inflige la désorganisation sociale à beaucoup de désorientés (ANGENOT, 1992, p. 12).

Angenot considère même qu'en élargissant «les idéologies du ressentiment $»^{2}$ aux particularismes, on pourrait dire que ces idéologies sont toutes des « régionalismes ». C'est donc à partir de ce moment qu'on peut affirmer que le tribalisme et le régionalisme

sont en effet des entités artificieusement créées; cela veut dire qu'activement l'idéologie du ressentiment cherche à contrer la fluidité sociale, à endiguer les interactions et interpénétration extérieures d'une part, et à dissimuler et nier l'hétérogénéité du groupe ressentimentiste même (Ibid, p. 19).

Ainsi, le tribalisme, le régionalisme et le ressentiment sont à l'œuvre dans le roman de l'écrivain-professeur Gabriel K. Fonkou. Chez lui, l'espace multiculturel donne lieu à un champ de positionnement où les individus «s'emploient constamment à se positionner à travers ce qu'ils disent, à s'affirmer en affirmant [...] à se valoriser

\footnotetext{
${ }^{2}$ Lire à ce sujet Angenot, Marc. Les idéologies du ressentiment. Essai. Montréal: XYZ Éditeur, 1996. (Prix «Spirale» de l’Essai 1996).
} 
et à surmonter les menaces de la dévalorisation » (Maingeneau, 1990, p. 18). Cela rend difficile voire impossible la coexistence entre les cultures. Gabriel K. Fonkou relève avec pertinence le caractère foncièrement conflictuel et ambivalent de ces lieux identitaires et montre la complexité des relations sociales. Cet article vise alors à montrer que dans Au pays de(s)intégrés l'ethnie est instrumentalisée et devient le moyen et le but suprême de toute action. Il sera question dans la présente étude de voir comment le tribalisme et le régionalisme sont des facteurs de désintégration. Nous essayerons aussi de montrer que le repli identitaire conduit aux constellations interculturelles.

\section{Tribalisme et régionalisme, moteurs de désintégration}

Au pays de(s)intégrés de Gabriel K. Fonkou relance la question de l'équilibre régional à travers les mésaventures d'un enfant naturel victime de tribalisme. La dédicace de roman résume parfaitement cette position «Aux enfants naturels de la tribu sans frontières ». Équilibre régional et intégration nationale peuvent-ils faire bon ménage ? Pour relancer le débat sur la politique des quotas, Gabriel Kuitche Fonkou a choisi de montrer ce qu'elle a de pervers à travers le parcours d'un enfant. Le titre de ce roman Au pays de(s)intégré(s) peut se lire «des intégrés » ou «désintégrés ». Gabriel K. Fonkou a joué avec les mots dans le titre de son œuvre pour se pose cette question fondamentale: "Sommes-nous au pays des intégrés ou au pays des désintégrés ? » Il relève les contradictions d'un État qui dit pourvoir l'intégration nationale, en même temps qu'il crée la désintégration, l'incompétence et l'injustice avec une horrible politique des quotas. De ce point de vue, Gabriel K. Fonkou laisse entendre :

On en parle toujours. Mais il me semble qu'il n'y a jamais eu dans ce pays autant de désintégration que depuis qu'on parle d'intégration. Alors que vaut le mot? Juste un mot. Un slogan désubstancialisé. Un paravent verbal derrière lequel se danse avec 
frénésie la danse de l'émiettement. Alors la réalité ? Un leurre. La conscience aiguë que n'implique pas toujours faire. La volonté sardonique d'entretenir en permanence le divorce entre la parole et les actes (KUITCHE FONKOU, 2013, p. 154).

La question de la construction nationale s'est toujours heurtée à la multiplication subtile des particularités ethniques, tribales et régionales. Ce phénomène discriminatoire fait que le cliché dichotomique artificiel «Nord-Sud», «Est-Ouest » devient une catégorie sociologique dont il faut nécessairement tenir compte dans la vie nationale et la notion vague d' « équilibre national » a fini par devenir un tremplin incontournable dans la vie politique, économique, sociale et culturelle. À ce propos, nous pensons à la suite de Denis Manière que le tribalisme en tant qu'une idéologie s'entend au sens marxiste de

système global d'idées, de concepts, d'images, de mythes et représentations sociales lié à un groupe économique, politique, ethnique justifiant des intérêts plus ou moins conscients et égoïstes, c'est aussi une incitation à agir dans telle ou telle direction en fonction d'un jugement de valeur (1997, p. 13).

Une telle vision viscéralement vile a pour conséquences de polluer profondément les rapports sociaux entre les citoyens et d'entraver gravement l'épanouissement collectif sur le plan socioéconomique et socioculturel en entretenant des idées reçues, des préjugés et des non-dits. On le voit bien, la persistance du tribalisme moderne sous forme de non-dits et de discrimination tacite dans la vie politique et sociale provient de l'entretien souvent même inconscient de ces idées reçues et de ces préjugés nés des stéréotypes : 
- À cause des préjugés. Les parents de ma mère, des montagnards, ne voulaient pas d'un gendre «nkwa' ${ }^{3}$, de surcroît mangeur d'escargot et de chenilles.

- Ceux de ma mère, son père surtout, ne voulaient, ne veulent pas d'un montagnard pour gendre. Et même s'ils changeaient d'avis aujourd'hui, ma mère est déjà depuis des années mariées à quelqu'un d'autre.

- Quel fléau que ces préjugés ! Il ne causerait pas tant de ravages si nos grands-parents avaient pu, comme nous aujourd'hui, fréquenter le G.L.C. (KUITCHE FONKOU, 2013, p. 72).

On assiste ainsi à l'érection de «frontières » et de «barrières » visibles et invisibles entre êtres humains partageant pourtant le même espace vital. Dans ce sens, on peut affirmer avec Marc Angenot que « les individus, dés-illusionnés, tendent à se rallier à des drapeaux d'identités rancunières » (ANGENOT, 1992, p. 11).

L'usage démagogique fait de la notion de «tribu» et de la notion d'appartenance tribale conduit à l'émergence d'une idéologie nombriliste et destructrice connue sous l'appellation de tribalisme. On ne saurait parler de tribalisme sans parler de régionalisme. Dès lors, le tribalisme et le régionalisme se résument ainsi à «une friperie identitaire et un étal de maniérisme revendicateur » (Ibid.). Le régionalisme exprime la préférence arbitraire accordée à une région sans doute occupée par sa propre tribu au détriment des autres régions. Il renvoie au repli identitaire focalisé sur sa propre région de naissance ou d'adoption, en déviant aux autres régions leur droit de s'épanouir sainement sur tous les plans :

Tu ne sais rien n'est-ce pas? Eh bien moi, je vais vous le dire. Je suis allé aux renseignements. Ce garçon s'appelle Chónet. Ça veut dire qu'il est montagnard. Un tel nom ne peut venir que des montagnes. Moi, je ne veux rien avoir à faire avec un montagnard. Rien. Vous entendez ? Rien (KUITCHE FONKOU, 2013, p. 23).

\footnotetext{
${ }^{3}$ La note de l'auteur précise : « Nwa' : les Bamiléké appellent ainsi les Camerounais des autres tribus surtout les originaires du Littoral, du Centre, du Sud et de l'Est ».
} 
Le tribalisme quant-à lui renvoie à un lieu symbolique, souvent spirituel et quasi-indissociable existant entre un individu et une localité. Qu'on en juge :

Après les achats qu'il venait d'effectuer, Moundjoa aurait pu attendre dans la sérénité la plus totale l'entrée au collège de Zal Moundjoa. À cette époque dans cette région, les familles n'avaient pas de soucis à se faire pour les fournitures scolaires. L'État pourvoyait les élèves en tout, absolument en tout. Conçue comme une mesure d'incitation à l'endroit des défavorisés, des retardés scolaires, cette sollicitude des pouvoirs publics était appliquée avec discrimination: on admettait très difficilement dans les établissements scolaires les enfants en provenance d'autres régions. Des fonctionnaires, affectés ici malgré eux, avaient été quelques fois rattrapés in extremis dans les aéroports ou les gares routières d'où, par réaction contre le refus d'admettre leurs enfants à l'école, ils voulaient regagner des régions plus hospitalières (KUITCHE FONKOU, 2013, p. 41).

C'est dans cette logique qu'on peut comprendre Marc Angenot lorsqu'il déclare que le tribalisme est « une raison d'être et un mandat social qui permettent cependant de ne jamais sortir de soi-même. [Il] détermine une sorte de privation des universaux éthiques et civiques, un détournement ethno-égotiste des valeurs » (ANGENOT, 1992, p. 7). Le tribalisme et le régionalisme exaltent simplement, de ce point de vue, la glorification nombriliste d'une localité. Au pays de(s)intégré(s) illustre à merveille ce tribalisme exacerbé et ce nombriliste tribal. Ils se traduisent par une cohabitation tumultueuse et belliqueuse : «J'en ai éprouvé davantage de ressentiment envers les montagnards et les hommes en robes, surtout envers les montagnards, dont le succès mettait ainsi à nu notre nullité »(KUITCHE FONKOU, p. 37). Le tribalisme participe donc de ce que Marc Angenot appelle «les idéologies du ressentiment» (1992, p. 7). Le ressentiment, de l'avis de cet auteur, est donc « tout avantage, toute valeur dont on est frustré et dont d'autres sont possesseurs (usurpateurs) ou 
simplement usufruitiers, perçus comme privilège injustifiable, comme dol manifeste, comme préjudice commis à vos dépens » (Ibid). Tandis que dans Au pays de $(s)$ intégré $(s)$ certaines «frontières » et «barrières » tribalistes et régionalistes sont quasi-concrètes telles que les délimitations géographiques propres aux terroirs et aux entités ethniques et tribales, d'autres sont purement imaginaires et relèvent des phantasmes collectifs nés des préjugés, des idées reçues et des non-dits.

Quelques-unes des formes subtiles du tribalisme et du régionalisme dans $A u$ pays de(s)intégré(s) sont entre autres le refus doux et poli des mariages interethniques et intertribaux. Dans ce roman de Gabriel K. Fonkou, les mariages interethniques ne sont plus vus en tant qu'échanges intercommunautaires et en tant qu'enrichissements mutuels entre les communautés ethniques. On assiste soit à un mariage interethnique « superficiel » empreint parfois de préjugés non dissipés soit à un repli identitaire dans les questions matrimoniales :

- Tu ne te trompes pas, mais...

- Je crois que tu en veux toujours à mon père. Pourtant Sita Mpot a fini par épouser quelqu'un qui n'est pas de chez nous. Pourquoi n'as-tu pas chassé celui-là aussi ? Pourquoi n'as-tu pas confisqué ses enfants ?

- Ce n'est pas la même chose. Cet autre homme, que je ne vois du reste pas beaucoup non plus, n'est pas un montagnard. C'est tout (KUITCHE FONKOU, 2013, p. 90).

La fonction unificatrice et intégrative du mariage interethnique propre aux communautés ethniques est alors en train de se perdre au profit d'un modèle matrimonial égocentrique incapable de léguer aux générations futures des valeurs sociales sûres telles que la solidarité, la complémentarité et le respect mutuel intercommunautaire. Dans ces conditions, on peut comprendre Marie-Rose AbomoMaurin lorsqu'elle affirme :

Rien d'étonnant dès lors qu'à l'intérieur du même pays des clichés perdurent, que des rivalités voient le jour et encouragent, dans une 
certaine mesure, le tribalisme. L'autre, dans le même territoire national, ne peut être « soi » et mérite d'appellation d' « étranger », c'est-à-dire celui dont on se méfie et qu'on méprise, quand bien même il ressort de la même aire géographique. La métonymie devient alors la figure principale par laquelle l'individu désigne sa région. Ce regard nourrit un discours à la fois idéologique et ségrégationniste (2011, p. 70).

Bien plus, on assiste à un refus de reconnaissance des valeurs culturelles et sociales des autres communautés ethniques. La cacophonie sociale et le chaos politique ont entraîné deux phénomènes inquiétants que sont l'atrophie culturelle et le déracinement socioculturel à cause du tribalisme et du régionalisme. Ces deux phénomènes dangereux et très subtils se manifestent alors à travers une « reconstruction identitaire tribaliste » des différentes ethnies en s'appuyant sur la logique d'exclusion et à travers aussi un rejet de ses propres valeurs sociales et ancestrales.

Ainsi, la plus grave menace ethnocentriste et tribaliste pour la cohésion et la survie d'une société pluri-ethnique est l'instrumentalisation démagogique de la «concentration ethnique » qu'on peut remarquer au sein des corps socioprofessionnels :

Un désaveu aussi éloquent ne découragea point le major. Il tenta de créer un front des anciens. Échec. Il crut alors qu'il aurait plus de chance en créant un front de la tribu. À cet effet, il invita à prendre un pot les éléments du personnel qu'il estimait proche de lui. Pour en rendre le nombre significatif, il associa à la tribu pure tous ceux et toutes celles qui s'en rapprochaient par alliance ou par intercompréhension linguistique. [...] Alors qu'il était chef, que serait devenu le major si une fraction du personnel, tous les venantsvenants par exemple, s'était arrangée pour lui mettre des bâtons dans les roues? Quelle place le major fait-il à l'intégration nationale qu'appelle le discours politique ? (KUITCHE FONKOU, 2013, pp. 139-141) 
Dans cette perspective, l'idéologie tribaliste substitue au "pôle de modernisation-dépassement [...] un pôle de repli-ressentiment »(ANGENOT, 1992, p. 9) et le tribalisme « semble donner raison aux groupuscules qui, enfermés dans leur contentieux, proclament aussi «à chacun sa vérité »»(Ibid, p. 12). Il importe d'ajouter ici que la tribalisation de la sphère professionnelle et publique se lit aussi à travers les postes de nomination :

Notre province regorge de valeureuses ressources humaines, susceptibles d'apporter leur concours à la construction nationale dans plus de postes ministériels, dans les secrétariats généraux des ministères, dans les présidences des conseils d'administration et les directions générales des sociétés, les délégations provinciales, et que sais-je encore! [...] Nous émettons avec ferveur le vœu que désormais, il nous soit donné, à l'instar des autres provinces, de jouir de toutes les prérogatives d'une province à part entière et non d'une province à part. Que tout dans la République soit pour tous, à l'instar du soleil qui brille pour tout le monde bien qu'il se lève à l'est. Maintenant que notre frère se trouve aussi dans la cour des grands, nous comptons sur lui pour rappeler à qui de droit, à temps et à contretemps, cette vérité fondamentale (KUITCHE FONKOU, 2013, p. 133).

Ce faisant, la société ne donne plus au bonheur le visage de l'ordre ou de l'harmonie sociale. Ce qui va à l'encontre d'Aristote lorsqu'il pense que « ce n'est pas simplement en vue de vivre, mais plutôt en vue d'une vie heureuse qu'on s'assemble en une cité » (ARISTOTE, 384-322 avant Jésus-Christ). Dès lors, le tribalisme devient dans le roman de Gabriel K. Fonkou, « une habitude de l'esprit, une manière de ressentir, un mode de vie ».

En somme, le tribalisme et le régionalisme constituent des blocages qui conduisent à graves menaces pour la cohabitation harmonieuse des entités ethniques et aboutissent à une sérieuse pollution de la vie politique, économique et sociale au 
plan national. Ils forment, en effet, un terreau fertile pour la manipulation mentale dangereuse et l'embrigadement psychosociologique suicidaire des citoyens par des idéologies de ressentiment sans scrupule et des politiciens sans vergogne qui veulent simplement utiliser leurs concitoyens comme de simples objets et de simples moyens pour atteindre et satisfaire leur propre fin de domination égoïste. On assiste là à un véritable «tribalisme politique $»^{4}$.

\section{Du repli identitaire aux constellations interculturelles.}

Nous avons précédemment montré que le roman de Gabriel Kuitche Fonkou laisse transparaitre un univers manichéen dans lequel interagissent les cultures. De ce fait, le regard porté sur les «montagnards », on l'a démontré, est fortement teinté d'idées reçues et conduit, de facto, à des phénomènes d'hiérarchisation et d'exclusion. Bien plus, on pourrait parler de «l'ethnico-[tribal] et du relationnel ». Ethnico-tribal parce qu'à en croire Tandia, « l'acte de nomination de l'autre, [du montagnard] est fortement influencé par sa carte d'identité [tribale] et relationnel du moment où certains hétérodésignants témoignent à suffisance de l'opacité ou de la transparence du même à l'autre (2003b, p. 55).

Dans un tel contexte, l'espace multiculturel devient le théâtre d'un champ de positionnement ; ce qui rend difficile la coexistence entre les cultures ${ }^{5}$. Ce faisant, $A u$ pays de(s) intégré(s) met en scène les enjeux et logiques identitaires en république de Mécarénésie. Ainsi, Gabriel Kuitche révèle le caractère foncièrement conflictuel et ambivalent de ces lieux identitaires et montre la complexité des relations sociales qui se jouent à la croisée des appartenances, avant tout, ethniques et socioéconomiques.

\footnotetext{
${ }^{4}$ Nous empruntons l'expression «tribalisme politique» à Lonsdale, John, Ehnicité, morale et tribalisme politique, Londres, SAS, Trinity College, Cambridge, Traduit de l'anglais par C. Abel.

${ }^{5}$ Hans-Jürgen Lüsebrink distingue trois modèles de sociétés multiculturelles : le modèle d'apartheid, le modèle assimilationniste et le modèle polycentrique. Au regard du fait que les «montagnards » font l'objet de discrimination, on peut dire que la socialité multiculturelle du texte donne à voir un modèle d'apartheid ou ségrégationniste. De ce point de vue, l'intégration comme processus sociologique permettrait de briser l'exclusion et la séparation tout en menant à la formation d'une valeur commune c'est-à-dire à un modèle polycentrique. Voir pour cela Hans-Jürgen, Lüsebrink (2005) Interkulturelle Kommunikation, Interaktion, Fremdwahrnehmung, Kulturtransfer, Stuttgart, Metzler. Ici page 17.
} 
Alors, il apparait que l'ethnie est instrumentalisée et devient, du coup, l'objet principal de la loyauté, le but et le moyen de toute action ${ }^{6}$.

Toutefois, en allant à l'encontre des clichés comme obstacle à une harmonie interethnique, l'auteur de Au pays de(s) intégré(s) montre qu'à côté d'une société bâtie sur le mode de la répulsion, d'autres possibilités de dissoudre ces oppositions sont en gestation. C'est dans cette perspective que le narrateur procède à une déconstruction et au recentrage de la notion d'ethnie qui est source de distinction et de différenciation. D'ailleurs, dans la même lancée, Sita Mpot invite son père Pepa Moundjoua, figure emblématique du rejet, à faire preuve d'ouverture et d'acception des « montagnards ». À ce titre, elle déclare :

Les Montagnards sont pratiquement chez eux ici, installés depuis des dizaines et des dizaines d'années. Leurs enfants nés ici ont grandi avec nous, avec les nôtres, sont depuis devenus eux-mêmes des adultes. Nous vivons avec ces gens sans friction d'aucune sorte. Ils ont épousé des filles de chez nous, des garçons aussi. Ils sont intégrés..... (KUITCHE FONKOU, 2013, pp. 24-25).

La prise de parole de Sita Mpot vise à promouvoir une vision positive des différences individuelles et/ou culturelles, à déconstruire et à détruire l'homogénéité des cultures. D’une manière générale, elle prône la reconnaissance des savoirs et des pratiques d'ailleurs et s'inscrit dans la logique d'une rencontre culturelle qui comme le stipule Paul Ricœur (1990, p. 213), « [...] affirme l'existence d'ensembles culturels fortement constitués dont l'identité, la spécificité et la logique interne doivent être reconnues, mais qui ne sont pas entièrement étrangers les uns les autres tout en étant différents les uns des autres ». Plus loin, le narrateur montre que la fixation des cultures

\footnotetext{
${ }^{6}$ David Simo parle d'ethnicisation pour désigner toute pratique qui consiste dans la transformation de l'ethnie en une catégorie politique centrale, ce qui entraine la mobilisation des ressources publiques à son profit exclusif ou préférentiel ainsi que la discrimination d'autres groupes. L'ethnicisation, selon Simo, privilégie le niveau vertical donné d'intégration, la déclare absolu et nie toute possibilité d'intégration dans un ensemble grand. Pour plus d'amples informations consulter David, Simo (2006) « Elites et constructions identitaires : le piège tribal». In : David Simo (sous la direction de) Constructions identitaires en Afrique : enjeux, stratégies et conséquences, Yaoundé : Clé. Page 26-43. Notamment à la page 33 .
} 
en termes d'opposition relève d'une gageure. A contrario, il postule une interaction entre Soi et l'Autre non plus en termes d'identification et d'altérisation, mais comme étant une dynamique régit par des rapports de négociation et d'échanges puisque, selon l'expression de Paul Ricœur (Ibid, p. 12), « le soi-même implique l'altérité à un degré si intime que l'un ne se laisse pas penser sans l'autre [...] ». De ce point de vue, la remarque que fait Zal Moundjoua lors du voyage qui le mène de son Bagbet natal pour la capitale Mécarénésienne se situe à contre-courant de l'image d'une certaine transparence interethnique caractérisée par l'impossibilité du dialogue interculturel ${ }^{7}$ et d'une intégration. Si par «la force du préjugé» ${ }^{8}$ les tribus sont catégorisées, l'expérience que le héros de Kuitche fait de l'ethnie Bibidis, pourtant qualifiée d'anthropophages, témoigne d'une volonté affichée et affirmée de ne plus percevoir les relations interculturelles uniquement sous le prisme de la désintégration. Le narrateur rapporte :

Le courrier postal s'immobilisa devant le bureau de poste de Zamis, en pays Bibidis. Zal Moundjoa s'attendait à lire sur les visages des gens des signes de cannibalisme. Il n'en fut rien. La foule de vendeurs et vendeuses qui prit d'assaut le mastodonte, proposant, comme dans les arrêts antérieurs, une gamme variée de produits, n'affichait aucune marque distinctive. Deux vendeuses serrées contre le véhicule au niveau de la fenêtre de Zal Moundjoa, surprirent ce dernier en parlant à la perfection sa langue à lui. [...] Les Bibidis ne pouvaient pas être aussi mauvais qu'on voulait les présenter. Sinon que feraient dans leur pays des gens originaires de Bagbet? (KUITCHE FONKOU, 2013, p. 55).

\footnotetext{
${ }^{7}$ Le dialogue interculturel est défini par Claude Clanet comme étant «l'ensemble des processus psychiques, relationnels, groupaux, institutionnels générés par des interactions de cultures, dans un rapport d'échanges réciproques et dans une perspective de sauvegarde d'une identité culturelle des partenaires en relation. » Voir à ce sujet Claude, Clanet (1990) L'interculturel : Introduction aux approches interculturelles en Education et en Sciences Humaines, Toulouse, Presses universitaires du Mirail.

${ }^{8}$ Nous empruntons cette expression à Pierre-André Taguieff (1987) La force du préjugé : essai sur le racisme et ses doubles, Paris, La découverte.
} 
On le voit, le roman de Gabriel Kuitche Fonkou est foncièrement porté par un désir de réconciliation et de recollage des hiatus culturels. Par la même occasion, l'auteur de Moi taximan donne un coup de pied dans la fourmilière des idées reçues à travers un contre-discours qui se donne à lire comme une volonté de s'attaquer aux poncifs et d'aller au-delà de l'ethnocentrisme et du repli sur soi.

Dans le même sillage, le Grand Lycée de la Capitale Mécarénésienne constitue un véritable laboratoire interculturel où sont inculqués aux apprenants les valeurs de fraternité et d'acceptation réciproque indépendamment des origines diverses. C'est à juste titre que, prenant la parole le premier jour de la rentrée scolaire, le proviseur tient un discours qui déjoue les logiques disjonctives. Il affirme :

Il me plait de le rappeler aux anciens et de le dire aux nouveaux, le G.L.C est, comme toute école, une seconde famille, mais plus encore, un creuset, un moule spécial où se forge la conscience nationale. Vous entrez ici étant de tribus diverses, vous repartirez dans quelques mois, dans un an, dans quatre ans comme dans sept ans, peu importe en somme le temps passé chez nous, transformés en Mécarénésiens, ce qui n'est pas peu dire (KUITCHE FONKOU, 2013, p. 68).

Loin d'esthétiser un schisme culturel, Au pays de(s) intégré(s) met en relief un syncrétisme entre les systèmes culturels. En outre, Zal Moundjoa devenu Naoussi Chónet se voit affecté, après sa formation en médecine, dans une province étrangère. Quelques jours après sa prise de fonction, il rassemble tout son personnel en séance de travail au cours de laquelle il les invite à mettre de côté toutes les barrières culturelles afin de lutter contre l'ennemi commun qui n'est rien d'autre que la maladie. Il fait prévaloir ceci:

Ma conviction profonde est que nous avons à combattre un seul ennemi commun, la maladie. La maladie n'a pas de tribu, pas de nationalité, pas d'âge, pas de sexe, pas de couleur politique. La maladie c'est tout simplement la maladie. Et notre seule raison 
d'être, c'est de lutter contre elle, chacun avec tout son savoir et tout son savoir-faire, toute sa sollicitude, toute sa disponibilité, tout son esprit de solidarité, à l'exclusion de toutes autres considérations (KUITCHE FONKOU, 2013, p. 137).

Les excès bellicistes et nombrilistes sont bannis de la médiation interculturelle de Chónet puisqu'il se situe dans la recherche de ce Paul Ricœur appelle « l'universel régulateur » (1990, p. 213). Il faut tourner le dos à toutes les types de discriminations. À la question de Mâ Apouri qui lui demande s'il ne soignera que les personnes de sa tribu, Chónet répond par l'interrogative en demandant à cette dernière : «Quelle est ma tribu ?» (Idem, p. 121) Pour lui, tout comme pour Sévérin-Cécile Abega, «La tribu n'existe pas $»^{9}$.

Sur un tout autre plan, le narrateur de Kuitche montre que les clivages ne sauraient être réduits qu'au moyen de sacrifices extrêmement douloureux et violents c'est-à-dire à la capacité qu'auront les Mécarénésiens non seulement à se défaire des discours disjonctifs et mobilisateurs, mais aussi à faire preuve d'ethnorelativisme. Toutefois, cette démarche exclut toutes formes d'acculturation, car pour le narrateur, «certes l'intégration harmonieuse suppose des concessions faites les uns aux autres, mais elle n'exige pas le reniement de soi-même » (KUITCHE FONKOU, p. 151). Ainsi, l'intégration procède d'un processus ambivalent bâtie sur ce qu'Antony Kwamé Appiah appelle «Le cosmopolitisme enraciné » ${ }^{10}$, c'est-à-dire le fait de s'ouvrir à l'Autre en restant Soi-même.

Au final, le parcours initiatique de Zal Moundjoua ; autrement dit le processus heuristique de son identité le conduit à avoir trois familles : «Zal Moundjoua avait désormais trois familles : celle de Bagbet, celle de la capitale économique, celle de la capitale politique. Trois pères, trois mères, trois séries de frères et sœurs » (KUITCHE FONKOU, p. 84). Ces familles disséminées, on le constate, dans des lieux bien distincts sont, respectivement, représentées par Pepa Moundjoua, Sita Mpot et

\footnotetext{
${ }^{9}$ Sévérin-Cécile Abega (2006) «La tribu n'existe pas. » In : David, Simo (sous la direction de) Constructions identitaires en Afrique : enjeux, stratégies et conséquences, Yaoundé : Clé. Pages 71-83. ${ }^{10}$ Lire à ce sujet Anthony Kwamé Appiah (2005), The Ethics of Identity, Princeton, Princeton University Press.
} 
Chónet. Cette triangulation permet à Zal de se construire une identité interculturelle, dont le but est, selon Pierre Kodjio Nenguié, « la mise en scène et la construction des modèles d'identités syncrétiques [...] qui illustrent la déconstruction des replis identitaires ethnocentriques »(2004, p. 122). La reconstruction identitaire de Zal Moundjoua, on peut le constater, oppose, selon les propos de Jean-Loup Amselle, la « raison ethnologique » qui consiste à séparer, à classer, à catégoriser et à présenter les cultures comme des entités [différentes], à la « logique métisse $»^{11}$ qui renvoie à un processus d'interfécondation entre les cultures et qui met l'accent sur « l'indistinction ou le syncrétisme originaire » (Ibid, p. 9).

Au regard de ce qui précède, la (re)configuration identitaire de Zal Moundjoa fait tomber les différences et mène les entités culturelles vers ce qu'Édouard Glissant appelle le « rassemblement des convergences culturelles » (GLISSANT, 1997, p. 37). Ainsi, nous pouvons, pour ce qui est de Au pays de(s) intégré(s), dire avec HansJürgen Lüsebrink que c'est peut-être Claude Lévi-Strauss qui, de manière à la fois claire et provocatrice, a insisté sur le fait que la dimension conflictuelle, loin de se situer dans les marges du dialogue interculturel, se trouve au contraire dans son centre même, étant corrélative de toute volonté d'affirmation de soi et de maintien de la propre identité:

Que conclure de tout cela, sinon qu'il est souhaitable que les cultures se maintiennent diverses, ou qu'elles se renouvellent dans la diversité? Seulement [...] il faut consentir à en payer le prix: à savoir, que des cultures attachées chacune à un style de vie, à un système de valeurs, veillent sur leurs particularismes; et que cette disposition est saine, nullement - comme on voudrait nous le faire croire pathologique. Chaque culture se développe grâce à ses échanges avec d'autres cultures. Mais il faut que chacune y mette une certaine résistance, sinon, très vite, elle n'aurait plus rien qui lui appartienne en propre à échanger. L'absence et l'excès de communication ont l'un

${ }^{11}$ Lire à ce propos Jean-Loup Amselle (1990), Logiques métisses: Anthropologie de l'identité en Afrique et Ailleurs, Paris, Payot. pp. 9-10. 
et l'autre leur danger (LÉVI-STRAUSS, cité par Lüsebrink, 1997, p. $30)$.

\section{CONCLUSION}

$\mathrm{Au}$ total, il était question dans la présente analyse de montrer qu'Au pays de(s)intégré(s) de Gabriel K. Fonkou est un espace multiculturel et pluri-ethnique dans lequel la coexistence entre les cultures et les peuples est malheureusement difficile et même presque impossible. Ce roman est le théâtre des conflits tribaux, ethniques et régionalistes qui complexifient la communication et la compréhension entre les peuples partageant pourtant le même espace vital. Gabriel K. Fonkou a ainsi cherché à fabriquer un modèle culturel et identitaire qui brise toutes les barrières ethnicotribales. Toutefois, cette tentative d'associations interculturelles échoue devant certaines pontes tribales et régionalistes parce que le ressentiment comme corollaire du tribalisme «est ce qui soude la tribu dont l'identité-cohésion ne résulte que du ressassement collectif de griefs et de rancunes » (Angenot, $1992: 19$ ). On peut finalement affirmer que pour ce qui est de $A u$ pays de(s)intégré(s) la dimension conflictuelle et donc tribale des cultures ne peut se situer dans les marges du dialogue interculturel. Elle doit se trouver au cœur de ses préoccupations même ; ce qui revient à dire que les cultures peuvent se maintenir diverses, mais se renouvellent dans la diversité. 
ABEGA, Sévérin-Cécile. « La tribu n'existe pas ». In : SIMO, David. (Sous la direction de) Constructions identitaires en Afrique: enjeux, stratégies et conséquences. Yaoundé, Clé, 2006, pp.71-83.

ABOMO-MAURIN, Marie-Rose. «Territoire national et altérité : de l'appartenance régionale géo-physique à l'exclusion ou l'utopique unité nationale ». In : ALBERT, Christiane ; ABOMO-MAURIN ; Marie-Rose, GARNIER ;Xavier et PRIGNITZ, Gisèle. (Sous la direction de). Littératures africaines et territoires. Paris : Karthala, 2011, pp.69-78.

AMSELLE, Jean-Loup. Logiques métisses : Anthropologie de l'identité en Afrique et Ailleurs. Paris : Payot, 1990.

ANGENOT, Marc. Les idéologies du ressentiment. Essai. Montréal: XYZ Éditeur, 1996. (Prix «Spirale» de l'Essai 1996).

ANGENOT, Marc. Le discours social, Volume 4, 3\$4, été-automne, 1992.

APPIAH, Anthony Kwamé. The Ethics of Identity. Princeton: Princeton University Press, 2005.

CAMILLERI, Carmel et COHEN-EMERIQUE, Margalit (Sous la direction de). Chocs de cultures : concepts et pratiques de l'interculturel. Paris : L'Harmattan, 1989. CLANET, Claude. L'interculturel: Introduction aux approches interculturelles en Education et en Sciences Humaines. Toulouse : Presses universitaires du Mirail, 1990. GLISSANT, Edouard. Traité du Tout-monde. Paris : Gallimard, 1997.

KODJIO NENGUIE, Pierre. «Interculturalité comme méthode et catégorie d'interprétation postmoderne : réflexions critiques et repères». In: Germanistik in und zwischen den Kulturen : Festschrift für David Simo zum 25 -jährigen Wirken an der Universität Yaoundé. Leipzig: Universitätsverlag, 2004, pp.112-126.

KUITCHE FONKOU, Gabriel. Au pays de(s) intégré(s). Cameroun : Éditions Clé en coédition avec NENA, 2013.

LONSDALE, John., Ehnicité, morale et tribalisme politique. Londres : SAS, Trinity College, Cambridge, 1996 (Traduit de l'anglais par C. Abel). 
LÜSEBRINK, Hans-Jürgen. Interkulturelle Kommunikation, Interaktion, Fremdwahrnehmung, Kulturtransfer. Stuttgart: Metzler, 2005.

LÜSEBRINK, Hans-Jürgen. «Domination culturelle et paroles résistantes. De la dimension conflictuelle dans la communication interculturelle ». In : Littérature et dialogue interculturel: culture française d'Amérique. Laval : Presses Université Laval, 1997, pp. 19-33.

MAINGUENEAU, Dominique. L'analyse du discours: introduction aux lectures de l'archive, Paris : Hachette, 1990.

MANIERE, Denis. Le Développement des idéologies au Québec des origines à nos jours. Montréal : Québec/Amérique, 1997.

RETSCHITZKY, J., BOSSEL-LAGOS, M., DASEN, P. (sous la direction de). La recherche interculturelle, tome 1, Paris : L'Harmattan, 1989.

RICEUR, Paul. Soi-même comme un autre. L'Ordre Philosophique, Paris : Seuil, 1990.

SIMO, David. «Elites et constructions identitaires : le piège tribal ». In : David Simo (sous la direction de) Constructions identitaires en Afrique: enjeux, stratégies et conséquences. Yaoundé : Clé, 2006, pp.26-43.

TAGUIEFF, Pierre-André. La force du préjugé : essai sur le racisme et ses doubles, Paris : La découverte, 1987.

TANDIA, MOUAFOU J-J.Rousseau. «Les «mêmes » face aux « autres » : 1'altérité déniée dans La Croix du Sud de Joseph Ngoué ». In : Sudlangues, n³, 2003a, pp. 53-69. «Au pays de(s) intégré(s) de Gabriel Kuitche Fonkou : pour une poétique de l'identité et de l'altérité Jean-Jacques Rousseau ». In PANGOP KAMENI, Alain Cyr et DILI PALAÏ, Clément (Sous la direction de). La création littéraire de Gabriel Kuitche Fonkou. Yaoundé : Éditions Clé, 2013b. pp.169-180. 